\title{
Splits in students' beliefs about learning classical and quantum physics
}

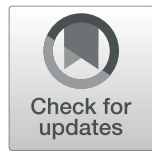

Benjamin W. Dreyfus ${ }^{1 * \dagger} \mathbb{D}$, Jessica R. Hoehn ${ }^{2 \dagger}$, Andrew Elby $^{3}$, Noah D. Finkelstein $^{2}$ and Ayush Gupta ${ }^{4}$

\begin{abstract}
Background: While there has been increasing recognition of the importance of attending to students' views about what counts as knowing and learning a STEM field, surveys that measure these "epistemological" beliefs are often used in ways that implicitly assume the fields, e.g., "physics," to be a single domain about which students might have sophisticated or naïve beliefs. We demonstrate this is not necessarily the case and argue for attending to possible differences in students' epistemological beliefs across different sub-domains of physics. In modern physics and quantum mechanics courses for engineering and physics students, we administered a set of modified Colorado Learning Attitudes about Science Survey (CLASS) items. Each selected item was turned into two items, with the word "physics" changed to "classical physics" in one and "quantum physics" in the other.

Results: We found significant splits between students' survey responses about classical vs. quantum physics on some items, both pre- and post-instruction. In classical physics, as compared to quantum physics, students were more likely to report the salience of real-world connections and the possibility of combining mathematical and conceptual reasoning during problem solving.

Conclusions: These findings suggest that attending to sub-domain specificity of students' beliefs about physics can be fruitful and ought to influence our instructional choices.
\end{abstract}

Keywords: Physics education research, Epistemology, Attitudes, Quantum physics

\section{Introduction}

Over the last decade or more, the physics education community has grown to acknowledge the importance of attending to students' views about what counts as knowing and learning physics (Elby, 2011; Madsen, McKagan, \& Sayre, 2015) - in the same way that students' views about knowing and learning are significant in other domains (Edmondson \& Novak, 1993; Rodríguez \& Cano, 2006). These views correlate with measures of conceptual learning (Adams et al., 2006; Brewe, Kramer, \& O’Brien, 2009), student performance in courses (Perkins, Adams, Pollock, Finkelstein, \& Wieman, 2005), and persistence in the major (Perkins, 2006). Not only do students' views about knowing and learning matter, but teachers' views are reflected in their teaching, both implicitly and explicitly; the environments that we help

\footnotetext{
* Correspondence: bdreyfu2@gmu.edu

${ }^{+}$Benjamin W. Dreyfus and Jessica R. Hoehn contributed equally to this work. 'Department of Physics and Astronomy/STEM Accelerator Program, George Mason University, Fairfax, VA 22030, USA

Full list of author information is available at the end of the article
}

construct as teachers impact students' learning (Linder, 1992; Marshall \& Linder, 2005; Tolhurst, 2007).

Much of this research around attitudes and beliefs toward physics has considered "physics" to be a single domain. This paper challenges this assumption and demonstrates that students can think differently about the nature of learning and knowing physics across different sub-domains of physics. In this study, students answered modified versions of selected items from the Colorado Learning Attitudes about Science Survey (CLASS) (Adams et al., 2006), distinguishing between "classical physics" and "quantum physics." We observed splits-differences between views about learning classical and quantum physics-in individual students' responses. Our study is exploratory in nature and demonstrates the existence of this phenomenon rather than pinpointing the breadth or mechanisms of the outcomes. These split CLASS results challenge various assumptions that we, as discipline-based education researchers and instructors, might make when attending to students' views about knowing and learning physics. We provide initial implications for research and for instruction and discuss 
further research directions that could address some of the questions raised here. These findings and questions are likely applicable across STEM disciplines more broadly, as other domains also have sub-field variation in content, approach, and application; here, we present one example in physics as a proof of concept of attending to sub-domain specificity of student views about learning.

\section{Debates about the coherence of epistemologies and what constitutes expertise}

We begin with a note about terminology. In this paper, we use the terms attitudes, epistemologies, and expectations. Attitudes broadly encompass opinions or emotional reactions to something (in this context, classical or quantum physics). This includes both epistemologies (views about the nature of knowledge and knowing) and expectations (views about what counts as knowledge or knowing for the purposes of doing well in school or a particular course). We situate our study within the physics education community that attends to all three of these constructs. For the present study, however, we focus primarily on the epistemology aspect. By "epistemology," we do not mean a branch of philosophy or our own methodological assumptions about how to generate valid knowledge as researchers. Rather, we use the term in the sense of "personal epistemology" (Hofer \& Pintrich, 1997), referring to individual students' beliefs about what counts as knowing and learning. The physics education literature commonly uses "epistemologies" in the plural, reflecting the diversity of epistemological views between students and within a single student.

This study contributes to two ongoing debates among researchers studying students' epistemologies and expectations (Elby, 2011). One debate addresses the extent to which students display general, domain-independent epistemological views about the nature of knowledge and knowing, or display different beliefs about different domains. Empirical work directly comparing students' views about, say, chemistry vs. psychology knowledge (see (Hofer, 2006) for review) has led to a growing consensus that at least some aspects of students' epistemological views are domain-specific, where "domain" in most of this research means an academic discipline (Greene, Sandoval, \& Bråten, 2016; Muis, Bendixen, \& Haerle, 2006). But researchers have left open the possibility that a "domain" need not correspond to a discipline. Our work adds to this line of research by demonstrating that students can exhibit different epistemological views about the classical physics encountered in first-year courses and the quantum physics encountered in third-semester and later courses. In other words, the "domains" of domain-specific epistemological views may be finer-grained than most previous literature has suggested.

A second debate to which we contribute concerns the nature of epistemological expertise/sophistication. In the broader survey- and interview-based literature on students' epistemologies of physics and science, researchers have defined expertise by scoring as more sophisticated the ideas that learning consists of active construction of ideas as opposed to absorption or memorization, that scientific knowledge is a product of human invention rather than read directly from nature, that knowledge is tentative rather than certain, that physics consists of interconnected concepts as opposed to just largely disconnected facts and equations, and that problem solving should involve conceptual and sometimes even intuitive ideas rather than just formulaic use of equations (Hammer, 1994; Hofer \& Pintrich, 1997; Redish, Saul, \& Steinberg, 1998). More recently, however, many researchers have argued that epistemological sophistication consists not of blanket assertions such as "knowledge is tentative," but rather, of contextualized judgments-e.g., explanations for why falling objects accelerate are much more certain than explanations for how black holes dissipate (Chinn, Buckland, \& Samarapungavan, 2011; Elby \& Hammer, 2001; Sandoval, 2005). If we conceptualize epistemological expertise as holding certain views at the expense of other views, then differences in students' attitudes toward classical and quantum physics might be interpreted as showing that students have less sophisticated epistemological beliefs about quantum physics than about classical physics. However, the notion that epistemological sophistication consists of the ability and propensity to make contextualized, nuanced judgments about knowledge and knowing opens up an alternative interpretation: students are judging quantum physics to be less intuitive and less tangibly connected to everyday experience, and quantum problem solving to rely more on mathematics and less on conceptual/intuitive reasoning, than is the case in introductory classical mechanics. Perhaps the recognition of these differences is itself a component of epistemological sophistication.

\section{Surveys of epistemological beliefs in physics}

A major thread in physics education research over the past two decades (Docktor \& Mestre, 2014) has focused on students' epistemological views about learning physics (Hammer, 1994). These beliefs have been assessed with a number of surveys, including the Maryland Physics Expectations Survey (MPEX) (Redish et al., 1998) and the Colorado Learning Attitudes about Science Survey (CLASS) (Adams et al., 2006). A student's response to each item is scored as being "favorable" or "unfavorable" (or "neutral"), and the student receives a score for each cluster/dimension (subset) of items into which the overall survey is divided (see (Adams et al., 2006) for details). For instance, CLASS results might be used to support the conclusion that students in a traditionally-taught physics class develop less expert-like views about physics than courses with an 
explicit focus on model building (Madsen et al., 2015). However, while these surveys break down the construct of expert-like beliefs about physics into multiple dimensions/ clusters, they treat the construct of "physics" itself as unitary. In this investigation, we explore the possibility that students display variability in their attitudes and beliefs about physics depending on the specific subfield within physics. But first, we briefly review prior work exploring context dependence in students' physics epistemologies.

\section{Context dependence in physics epistemologies unrelated to subfields of physics}

Most studies specifically designed to look for context dependence in students' epistemologies of physics have probed variability across activities and contextual features of problem presentation, not variability across subfields of physics. For instance, in a high school physics course, Muis and Gierus (2014) administered the same physics epistemology survey ("EBAPS home," n.d.) twice, a few weeks apart. In one condition, students took a conceptual physics test immediately before completing the epistemological survey. In the second condition, students instead took a more traditional procedurally-oriented physics test. All students participated in both conditions, in counterbalanced order. "Results revealed that girls espoused more constructivist beliefs about physics for conceptual knowledge than for procedural knowledge, whereas the opposite was found for boys" (Muis \& Gierus, 2014). So, this study found genderlinked differences in students' epistemologies of physics based on whether students had been primed by conceptual vs. "standard" physics problems.

Other studies have found other aspects of context dependence (though not gender-linked) within individual students' physics epistemologies, such as variation in views about the role of theory in data interpretation when probed in the abstract vs. in a specific experimental context (Leach, Millar, Ryder, \& Séré, 2000), and differences in a student's views about the role of intuitive or everyday knowledge when addressing physics problems in the classroom during group work vs. outside the classroom during an interview (Lising \& Elby, 2005). Our point is that, although context dependence in students' epistemologies of physics is fairly well-established, context dependence across subfields of physics has not been explored.

\section{Survey-based studies potentially bearing on subfield dependence of epistemologies}

Most published survey-based studies of student epistemologies/expectations report pre-post results in introductory physics courses, making it impossible to break down the results in terms of specific physics topics or subfields. A few longitudinal studies, however, may bear on subfield dependence of physics epistemologies.
For instance, Gire, Jones, and Price (2009) administered the CLASS to physics majors in all 4 years, and found no significant change in favorable responses during the first 3 years, but a shift toward expert-like views in year 4 . The courses in which the CLASS was given included quantum mechanics, but the analysis bins students by year rather than by course and does not address the issue of subfields. Bates, Galloway, Loptson, and Slaughter (2011) did a similar "pseudolongitudinal" study, giving the CLASS to cohorts from high school to physics faculty. Again, the analysis bins undergraduate students by year rather than by course. They observed a statistically significant decrease in favorable responses for undergraduates in year 3 and explain this result as anomalous. We wonder whether this is the year when most students were taking quantum mechanics, though there is not sufficient information in the paper to draw any conclusions about this.

McCaskey and Elby (2005) looked at the impact of the modern physics context. They found that one reformed introductory physics course demonstrated epistemological gains on the MPEX, while a similar reformed introductory course which included modern physics topics showed losses. There were other differences between the courses, so they could not conclude that modern physics was the relevant variable. However, when the professor of the latter course scaled back the modern physics coverage the following year, the course showed MPEX gains. McCaskey hypothesizes "that modern physics ideas are often hard to reconcile with experience. They can seem like disconnected pieces, especially when compared to previous parts of the course. Students may feel like modern physics ideas are wacky and require acceptance, and not reconciliation or sense-making." (McCaskey, 2009)

Finally, Mason and Singh (2010) gave the Attitudes and Approaches to Problem Solving (AAPS) survey to physics graduate students, asking them to complete the survey twice: once when thinking about problem-solving in their own graduate physics courses, and once when thinking about problem-solving in introductory physics. The responses about introductory courses were more expert-like, and the authors attributed this both to graduate students not yet being "experts" in graduatelevel physics and to the traditional instructional approaches used in graduate courses. Again, we wonder if graduate quantum classes may have contributed to the divergent results seen in this study.

Crucially, all of these studies score and interpret survey results in the manner defined in the original CLASS development (Adams et al., 2006), associating higher sophistication with the expert consensus favorable responses to questions that ask about "physics" as a whole. This is an approach we will challenge below by attending to sub-domain specificity of epistemologies; when we 
ask about classical and quantum physics separately, it may not make sense to continue to associate the favorable responses on the original CLASS with higher sophistication.

\section{Bifurcated surveys}

As noted above and discussed in detail below, our study uses a bifurcated survey, a survey in which each item from a previously existing survey is turned into two items. Our methodology is inspired and informed by several past physics education research (PER) studies involving bifurcated surveys. McCaskey (2009) gave students the force concept inventory (FCI) (Hestenes, Wells, \& Swackhamer, 1992), and asked them to give both the answer "you really believe" and the answer "you think scientists would give." The former prompt was later changed to "that makes the most intuitive sense to you." Splits were observed at the end of the course, suggesting that students had not reconciled their physics knowledge with their intuition. However, the splits were smaller in a reformed course promoting this kind of reconciliation. Gray et al. (2008) similarly bifurcated the CLASS, asking students to select both the choice "that best expresses your feeling" and "the choice that you think a physicist would give." They found that students correctly identified the "expert" views but did not personally agree with those views. This result was used to demonstrate both the validity of the CLASS, by showing that students were willing to express their own views instead of the "right answer," and the failure of some courses to help students see expert-like beliefs as useful.

McCaskey's study bifurcated a content survey (the FCI) along epistemological lines, while Gray et al.'s study bifurcated an epistemological survey (the CLASS) along epistemological lines. Our study occupies a third category, bifurcating an epistemological survey along content lines (classical vs. quantum). The most similar previous study is Mason and Singh's (2010), discussed above. We expand on that work in two ways. First, problem solving is one dimension that we examine, but we look at others as well. Second, while the graduate physics courses in Mason and Singh's study probably included quantum mechanics, our survey asks specifically about quantum versus classical physics.

\section{Goals of this study}

This investigation is part of a larger study of student thinking in quantum mechanics (Dreyfus, Elby, Gupta, \& Sohr, 2017; Hoehn \& Finkelstein, 2018b; Hoehn, Gifford, \& Finkelstein, 2019; Sohr, Gupta, \& Elby, 2018; Tutorials on thinking about quantum entities, n.d.). Our studies take place in two different levels of quantum mechanics (QM) instruction in undergraduate curriculum for physics majors in the US-a more general course typically called "Modern Physics" and more specialized advanced quantum mechanics. We focus primarily on the Modern Physics course. In the USA, this class typically includes special relativity and an introduction to QM ("modern" generally refers to physics from the early twentieth century); for physics majors, this precedes a more in-depth upper-level course on QM. Within the larger study, we observed anecdotally that students' attitudes about quantum mechanics differ in some ways from their attitudes towards physics in general. We designed and administered a bifurcated survey to address the following research question:

1. In the context of Modern Physics and quantum mechanics classes, are there differences in students' self-reported epistemological beliefs about quantum and classical physics?

To understand these quantitative results, we also use qualitative data to address a second research question:

2. Do Modern Physics students in interviews spontaneously express epistemological views related to differences between classical and quantum physics?

A "yes" answer to this question would enable us to interpret our bifurcated survey results as reflecting students' consciously held views, not just their tacit views that crystallize into survey responses shaped largely by the bifurcated structure of the survey itself. Future analysis of qualitative interview data will examine more deeply the specific reasons students provide for their views about the differences (or similarities) between quantum and classical physics.

We divide the "Methodology" section into two subsections: quantitative methods used to address research question (1) and the qualitative methods used to address research question (2).

\section{Methodology \\ Quantitative methods for research question 1: do classical-quantum epistemological splits exist? Data collection}

This section addresses the first research question: In the context of Modern Physics and quantum mechanics classes, are there differences in students' self-reported epistemological beliefs about quantum and classical physics? To investigate whether students report different epistemological beliefs about classical and quantum physics, we selected Likert-scale items from the CLASS, taken from multiple categories (Adams et al., 2006), and bifurcated them into classical and quantum versions. For example, the CLASS item "Knowledge in physics 
consists of many disconnected topics" was modified to "Knowledge in classical physics..." and "Knowledge in quantum physics...." One block of questions was introduced by the instruction, "For these questions, please think about your previous physics courses on motion, electrical phenomena, etc., which we are calling "classical' physics in the items below," and the other was introduced by, "For these questions, please think about past/upcoming courses on modern physics and/or quantum physics."

The CLASS assesses attitudes on a number of dimensions, and we were specifically looking at epistemological attitudes, so we did not include items such as "I enjoy solving physics problems." Using our instructional and researcher intuitions, not a systematic procedure, we further narrowed the survey and chose six CLASS items based on two factors: alignment with the epistemological agenda of the Modern Physics course at University of Colorado (CU) Boulder, and our predictions (based on experience with students) about the likelihood that students would "split" their responses. So, for instance, we anticipated that in comparison to classical physics, students may perceive quantum physics to be less tangibly connected to real-world experiences, less conceptually intuitive, and more needful of a "shut up and calculate" approach to problem solving. Some of these items (e.g., \#41) were also chosen because of prior work (Baily \& Finkelstein, 2009) that suggests that students' responses to these items may be influenced by the quantum context. Table 1 in the "Results" section below presents our six items. Our approach to selecting specific CLASS items is appropriate for this study, because our goal was to see if students displayed different epistemological views about classical and quantum physics at all (which could be established by splits on a single item), not to characterize the range and substance of the differences or to develop a new survey. Thus, in this exploratory study, we did not formally re-validate the bifurcated items. We did, however, check that students understood the questions in the way we intended and that their answers reflected their epistemological views rather than being a result of the structure of the survey itself. We assumed that (i) students' comments during interviews would provide insight into how they interpreted "classical" and "quantum," and (ii) given the validation studies on the original CLASS items, students would still know what the items mean when the word "physics" is replaced by "classical physics" or "quantum physics." Students' comments during interviews, as discussed in the "Results" section, did indeed suggest that they interpreted "quantum" and "classical" in the way we intended. Further, analysis of interviews suggests that students did spontaneously express differences in epistemological views of classical and quantum physics.

The bifurcated survey questions were administered in four different courses spanning two universities and four semesters: Modern Physics for Engineers at the University of Colorado Boulder (CU) in semesters 1 and 3 of the study, Modern Physics at the University of Maryland (UMD) in semester 2, and Quantum 1 at CU in semester 4. The Modern Physics course at CU is the third semester of the introductory physics sequence, enrolling primarily engineering students. It focuses on introductory quantum mechanics and emphasizes applications and interpretations of quantum mechanics (Baily \& Finkelstein, 2010). In semester 3 , the sole instructor from semester 1 was joined by a co-instructor. Though the semester 3 course used most of the original course materials, the co-teaching arrangement resulted in shifted emphases-less on applications and interpretations-compared to semester 1. UMD Modern Physics is an upper-level elective for engineering students that spends several weeks on special relativity and the rest of the semester on quantum mechanics. CU Quantum 1 is the first semester of upper division quantum mechanics for physics majors. In semester 4 , this course was taught with a spins-first pedagogical approach. Both CU and UMD are large, 4-year, public research institutions. We did not collect demographic information from the students who participated in our survey, but we report demographics at the school or department level (as available) to provide context for the study. The students enrolled in the semesters 1 and 3 courses at $\mathrm{CU}$ were predominantly engineering majors. The CU School of Engineering (as of Fall 2018) is 72\% male and $28 \%$ female and the racial demographics are as follows: $61 \%$ white, $12 \%$ Hispanic/Latino, $12 \%$ Asian, 10\% International, $2 \%$ African-American, $1 \%$ American Indian/Alaska Native, $0.6 \%$ Native Hawaiian/Pacific Islander, and $0.6 \%$ Unknown ("CU Boulder fall enrollment over timeBy college-University of Colorado Boulder IR | Tableau Public," n.d.). The students enrolled in the semester 4 course at $\mathrm{CU}$ were predominantly physics majors. The Physics Department at CU (as of Fall 2018) is 84\% male and $16 \%$ female and the racial demographics are as follows: $60 \%$ white, 18\% international, 9\% Hispanic/Latino, 7\% Asian, 3\% African-American, 2\% American Indian/Alaska Native, $0.3 \%$ Native Hawaiian/Pacific Islander, and 0.5\% Unknown ("CU Boulder fall enrollment over time-By major-University of Colorado Boulder IR | Tableau Public," n.d.). We do not have demographic information for the UMD Physics Department specifically, but the racial demographics of UMD overall are as follows: $49 \%$ white, $17 \%$ Asian, 12\% Black or African-American, 10\% Hispanic, 5\% Foreign, $4 \%$ two or more, $3 \%$ unknown, and $0.1 \%$ each American Indian/Alaska Native and Native Hawaiian/Pacific Islander (UMD Undergraduate Student Profile, 2018).

Students answered the bifurcated CLASS questions as part of a larger survey that also included some epistemological questions specific to quantum mechanics (Baily \& Finkelstein, 2009). For the Modern Physics courses in semesters $1-3$, the survey also contained some quantum mechanics conceptual questions taken from the 
Table 1 Classical/quantum splits on the bifurcated CLASS questions. The $p$ values are determined with the Bhapkar test and corrected using the Holm-Bonferroni method. Items with significant splits at the $a=0.05$ level are indicated in italic

CLASS Item

consists of many disconnected topics.

23: In doing a [classical/quantum] physics problem, if my calculation gives a result very different from what l'd expect, I'd trust the calculation rather than going back through the problem.

28: Learning [classical/quantum] physics changes my ideas about how the world works.

35: The subject of [classical/quantum] physics has little relation to what I experience in the real world.

40: If I get stuck on a [classical/ quantum] physics problem, there is no chance I'll figure it out on my own.

41: It is possible for physicists to carefully perform the same measurement in a [classical/ quantum] physics experiment and get two very different results that are both correct.

\begin{tabular}{|c|c|c|}
\hline Survey & $N$ & $p$ value \\
\hline Semester 1 pre & 118 & 0.00018 \\
\hline Semester 1 post & 114 & 0.58 \\
\hline Semester 2 pre & 21 & 0.171 \\
\hline Semester 2 post & 22 & 0.22 \\
\hline Semester 3 pre & 43 & 0.00004 \\
\hline Semester 3 post & 51 & 0.42876 \\
\hline Semester 4 post & 40 & 0.45479 \\
\hline Semester 1 pre & 118 & $<0.00005$ \\
\hline Semester 1 post & 113 & 0.0006 \\
\hline Semester 2 pre & 21 & 0.0015 \\
\hline Semester 2 post & 22 & 0.0282 \\
\hline Semester 3 pre & 43 & 0.0018 \\
\hline Semester 3 post & 51 & 0.00294 \\
\hline Semester 4 post & 40 & 0.00291 \\
\hline Semester 1 pre & 118 & 0.98 \\
\hline Semester 1 post & 108 & $<0.00005$ \\
\hline Semester 2 pre & 21 & Undefined \\
\hline Semester 2 post & 21 & 0.975 \\
\hline Semester 3 pre & 43 & 0.02824 \\
\hline Semester 3 post & 49 & 0.00036 \\
\hline Semester 4 post & 40 & 0.01141 \\
\hline Semester 1 pre & 117 & $<0.00005$ \\
\hline Semester 1 post & 108 & 0.0006 \\
\hline Semester 2 pre & 21 & 0.0291 \\
\hline Semester 2 post & 22 & 0.004 \\
\hline Semester 3 pre & 43 & 1 \\
\hline Semester 3 post & 51 & Undefined \\
\hline Semester 4 post & 40 & $8.93 E-09$ \\
\hline Semester 1 pre & 118 & $<0.00005$ \\
\hline Semester 1 post & 104 & $<0.00005$ \\
\hline Semester 2 pre & 21 & 0.0021 \\
\hline Semester 2 post & 22 & $<0.00005$ \\
\hline Semester 3 pre & 43 & 0.00004 \\
\hline Semester 3 post & 51 & 0.00015 \\
\hline Semester 4 post & 40 & 0.03518 \\
\hline Semester 1 pre & 118 & $<0.00005$ \\
\hline Semester 1 post & - & - \\
\hline Semester 2 pre & 21 & 0.0102 \\
\hline Semester 2 post & 22 & 0.00004 \\
\hline Semester 3 pre & 43 & 0.00004 \\
\hline Semester 3 post & 51 & 0.00004 \\
\hline Semester 4 post & 40 & 7.09E-18 \\
\hline
\end{tabular}


Quantum Mechanics Conceptual Survey (QMCS) (McKagan, Perkins, \& Wieman, 2010) and elsewhere.

\section{Data analysis}

In typical analyses of data from attitude surveys such as the CLASS, the survey items are grouped into clusters, and within each cluster, the possible responses to each item are scored as "favorable" or "unfavorable." In our case, scoring a bifurcated cluster of items would require making decisions about which response is "favorable" and having that decision apply to both the classical and quantum version of the item. In this study, we wanted to leave open the possibility of challenging precisely that assumption. Thus, rather than grouping items into clusters, we looked for classical/quantum splits on each individual bifurcated pair of items.

The survey items are on a 5-point Likert scale from Strongly Disagree to Strongly Agree. However, as is common in PER studies using Likert-scale data (Adams et al., 2006; Redish et al., 1998), we collapsed Strongly Agree and Agree into a single category, and likewise for Strongly Disagree and Disagree. This permits us to see only strong splits (e.g., between "agree" and "neutral," or between "agree" and "disagree"), not weak splits (e.g., between "strongly agree" and "agree"). We avoided treating the Likert scale-even the collapsed 3-point version-as interval data, where the points on the Likert scale are treated as uniformly spaced, making it possible to calculate an average score for each given item). We did this both because there is controversy in the literature about whether it is appropriate in general to treat Likert-scale data as interval data (Jamieson, 2004; Norman, 2010) and for the more specific reasons discussed by Adams et al. (2006): validation interviews for the CLASS showed that students had a variety of reasons for choosing Neutral, so we cannot assume that Neutral represents a point halfway between Agree and Disagree. Instead, we treated the Likert scale as ordinal data, where the options are discrete categories rather than points on a continuum.

Because ordinal data cannot be averaged, we could not use traditional statistical tests such as $t$ tests to determine whether the classical/quantum splits were statistically significant. Instead, we used the Bhapkar test (Bhapkar, 1966; Ireland, Ku, \& Kullback, 1969; Sun \& Yang, 2008), a generalization of the McNemar test (McNemar, 1947) to more than two categories. The McNemar test compares two dichotomous distributions to determine whether they are significantly different. It has been used in the PER literature (Ding, Chabay, \& Sherwood, 2013; Leinonen, Asikainen, \& Hirvonen, 2013; Stewart, 2007) to compare student performance on individual concept inventory items under different conditions (e.g., pre and post, or two versions of the same question), where the two categories were "correct" and "incorrect." However, our data set has three irreducible categories (Agree, Neutral, and Disagree), so we used the more general Bhapkar test. Notably, the Bhapkar test (like the McNemar test) requires knowing not only how many students gave each response (Agree, Neutral, and Disagree) under each condition (classical and quantum) but also requires filling a $3 \times 3$ matrix with each permutation of responses for a given student-how many students selected Agree for classical and Neutral for quantum, and so forth for the other eight permutations). Thus, the Bhapkar test "knows" how many students actually split their responses, and not only the population totals. The Bhapkar test is similar to the Stuart-Maxwell test (Maxwell, 1970; Stuart, 1955), and is asymptotically equivalent to it at large N, but is considered more powerful at smaller $\mathrm{N}$ (Keefe, 1982; Sun \& Yang, 2008).

To determine significant classical/quantum splits, we performed the Bhapkar test on each bifurcated CLASS item for each administration of the survey. Students who left either the classical or quantum question blank were excluded from the analysis for that item. The $n \times n$ Bhapkar test (where $n=3$ in our case) yields a chisquared statistic with $n-1$ degrees of freedom, which can then be converted into a $p$ value to determine significance.

One aspect of this methodology initially troubled us. The Bhapkar test treats Agree, Neutral, and Disagree the same way it would treat Vanilla, Chocolate, and Strawberry-as unordered categories. For the purposes of this study, however, it makes sense to think of Neutral as between Agree and Disagree. To determine whether this made a difference, we tried collapsing the six off-diagonal elements of the $3 \times 3$ matrix into two: "classical > quantum" (which includes classical-Agree/quantum-Neutral, classical-Agree/quantum-Disagree, and classical-Neutral/quantum-Disagree), and "quantum > classical" (which includes the other three permutations where the classical and quantum responses differ). We ran a standard $2 \times 2$ McNemar test on this reduced matrix, which is possible because the Bhapkar and McNemar tests ignore the diagonal elements. We found no difference in terms of which items showed statistically significant splits between these $2 \times 2 \mathrm{McNemar}$ results and the $3 \times 3$ Bhapkar results. Therefore, we report the results from the Bhapkar test, which is established in the literature, and not from our own ad hoc test.

We treated each semester of survey data as a separate data set, because the four groups had different student populations, resulting in different starting points on the pre-semester survey, and because the courses differed in content and instructional approach. Specifically, there was one upper division QM course vs. three modern physics courses; and three reform-oriented courses 
(though reformed to varying degrees) and one traditionally taught class. By treating the groups separately, we observed significant splits that may have been washed out inappropriately had the groups been combined. It may have made sense to combine data from semesters 1 and 3 because these came from the same course at $\mathrm{CU}$, with similar student populations. However, the semester 3 course was co-taught, resulting in different instructional emphases as described above. The fact that we obtained similar results from the two semesters, as shown below in the "Results" section, suggests that these splits are robust across different instructional contexts. That is, they are not necessarily the result of one instructor teaching the course in a specific way.

We used the Holm-Bonferroni correction method (Holm, 1979) to account for the fact that we ran many comparisons (Bhapkar tests) and hence were likely to obtain one or more "statistically significant" results $(p<.05)$ that are actually just fluctuations. For each bifurcated item, we performed four tests: comparing classical and quantum for both the pre- and post-survey administrations, and comparing pre and post for both the classical and quantum versions of the item. Because our main goal is to explore the existence of classical-quantum splits, not to explore how the targeted courses influenced students' views, we only report on the classical-quantum comparisons here; pre-post shifts are left for a future paper. Thus, keeping each semester's data separate and conducting our analysis item-by-item, we corrected each $p$ value resulting from the Bhapkar test for four comparisons. The $p$ values from semester 4 are not corrected since there was only a post survey that semester and thus we only performed one test on each item.

These tests measure statistical significance (whether or not there is a significant split) but do not measure effect size (the magnitude of the split), and unfortunately the standard measures of effect size are not well-equipped to address data of this nature (ordinal data where the effect is measured by splits rather than similarities). However, the claims of this paper (demonstrating the existence of splits) do not depend on effect sizes.

\section{Qualitative methods for research question 2: do students spontaneously express splits? \\ Data collection}

This section addresses the second research question: Do Modern Physics students in interviews spontaneously express epistemological views related to differences between classical and quantum physics? As one of the multiple qualitative data streams collected for the larger project, we individually interviewed seven students recruited from the $\mathrm{CU}$ Modern Physics course in semester 1, both mid-semester and near the end of the semester. For this paper, we analyzed the end-of-semester interviews. The purpose of these videotaped interviews was to check in with the students individually on their experiences in the Modern Physics course and in "focus groups" (essentially, group problem solving interviews) about the nature of quantum entities and related interpretational issues in which the interviewees had previously participated. The 30 -min semi-structured individual interviews often involved unprompted meta-level conversations about learning in $\mathrm{QM}$, which is why we chose to code them for this study. See the Appendix for the interview protocol. Crucially, the interviews did not explicitly address CLASS items.

Of the seven interview participants, there were six males and one female. Three of the students identified as white, one as Asian, one as Hispanic/Caucasian, one as Japanese/Irish/German, and one chose not to identify their race/ethnicity.

\section{Data analysis}

We transcribed the interviews and coded them for spontaneous epistemological comparisons between classical and quantum physics. From the Modern Physics course culture in which they were embedded as well as their previous experiences with the research team, the interviewees likely knew that we valued externalizing and reflecting on epistemological views as an important part of learning quantum physics. Still, the interviewer did not generally ask students to compare their learning in classical vs. quantum physics. So, we coded for spontaneous epistemological comparisons in this way.

Starting from the beginning of the interview, the coder identified Epistemological statements-ones that pertain to how ideas in quantum physics connect with everyday experiences and/or intuitions, or to how learning or problemsolving in quantum mechanics should be approached, and other issues in the space spanned by the six bifurcated CLASS items. Upon identifying an epistemological statement, we then coded for Comparison-whether the student positions the ideas/concepts/results/equations encountered when learning quantum in contrast to those encountered previously in physics. (Students' previous courses were entirely classical.)

Having identified an epistemological comparison, the coder then reviewed the previous few turns of conversation to determine if the comparison was spontaneous. The coder classified the preceding prompts as prompting or not prompting a reflection on the comparison. For instance, if an interviewee responds to a prompt such as, "How is learning QM different from previous courses?" by making an epistemological comparison, the comparison is not spontaneous. By contrast, suppose the interviewer asks, "What do you see as the role of math in QM?" and the interviewee responds by stating that math is more important in QM than in classical physics 
because QM is counterintuitive. We code that as a spontaneous epistemological comparison because the interviewer did not ask for a comparison. So, "spontaneous" does not mean "out of the blue." It just means that the student, uncoerced, can articulate an epistemological comparison between classical and quantum physics, indicating that our split survey results do not correspond entirely to tacit beliefs or to artifacts of the bifurcated survey design. Instead of using a second coder to increase the accountability of our coding-based analysis, we include in our "Results" section an example of a spontaneous epistemological comparison from each interviewee who (we claim) offered one.

\section{Results}

Bifurcated survey results: research question 1

Table 1 summarizes the survey results: we found statistically significant splits between the classical and quantum versions of most of the questions, in both pre and post administrations.

Note that Table 1 lists a few results as undefined or blank. For item 28, semester 2, pre, no students chose Disagree for classical and Neutral/Agree for quantum, and no students chose Disagree for quantum and Neutral/Agree for classical. For item 35, semester 3, post, every student responded Disagree for the classical version. In these instances, running the Bhapkar test would involve dividing by zero. We can understand this to mean that there was not a classical/ quantum split on this item. For item 41, the quantum version of the item did not appear in this form on the post survey in semester 1.

More detailed results, with the percentage of students who "split" in each possible permutation, are given in the Additional file 1. A condensed version of this information is displayed graphically in Fig. 1. There are separate graphs for each administration of the survey. Each bar graph shows "no split" (the percentage of students who gave the same responses for classical and quantum, on the 3-point Likert scale), "quantum < classical" (students who chose classical-Agree/quantum-Neutral, classical-Agree/quantum-Disagree, and classical-Neutral/ quantum-Disagree), and "quantum > classical" (students who chose the other three permutations where the classical and quantum responses differ). We discuss some of the specific results below in the "Discussion" section.

\section{Spontaneous epistemological comparisons: research question 2}

In brief, of the seven interviewed students, six expressed a spontaneous epistemological comparison, suggesting that the classical-quantum splits found in the survey results potentially correspond to something psychologically robust for students.
In the rest of this section, we present one spontaneous epistemological comparison from each of those six students. Student names below are all pseudonyms.

\section{Wei}

Wei initiated a segment of conversation by stating, "Physics 1 and 2 is just really straightforward and you ... can do the exams and homeworks really easy. But for quantum, after you understand what's going on and you see the questions, but you still really confused about it. So it's really changed the way to think about physics." The interviewer followed up by asking "So it's like changing the way you think about physics but then that sometimes doesn't match with doing the homeworks or exams, not like with your previous experience, right?" Wei responded, "After you know the formulas about the physics like on physics 1 and 2 ... you just plug them into the questions. And then for quantum you must understand what he's saying and what he's asking and you can know the formulas but you sometimes cannot understand what he's saying at you." We see Wei's first statement as spontaneously comparing classical physics courses (Physics 1 and 2) with the quantum course he's currently taking. When prompted for clarification, Wei implied that the distinction has an epistemological component: in Physics 1 and 2, Wei felt that knowing the formulas is sufficient for solving problems, whereas in quantum mechanics, Wei felt the need to also understand what is being said about the problem context.

\section{Jacob}

Responding to a prompt about the role of math in the QM class, Jacob says, "Some of the math that we would've gotten into would be so complex I feel like it would just take up a ton of time to try and understand and maybe make us more confused than we were earlier and just be really hard. But I don't know, I feel like I'm typically a math kid. I like the math parts of that stuff more, like in physics 2 and 1 I totally liked the math more. I thought it was easy and fun..." Jacob here compares the mathematics he encountered in classical physics, which was "easy and fun," to the math he encountered in the QM class, which was "complex" and "really hard." Crucially, from an epistemological perspective, Jacob says that investing the "ton of time" needed to understand some of the QM math would have risked making "us more confused"- a hindrance rather than an aid to understanding. For both Wei and Jacob, we acknowledge an alternative interpretation of the data suggesting that the epistemological comparisons they are making are not primarily about classical vs. quantum physics, but about the way math is used in introductory vs. middle-division courses (such that they could have made similar comparisons in intermediate classical mechanics courses). While this may be present as an additional factor, the data from the other students (presented below) bring in additional factors that are specific to 

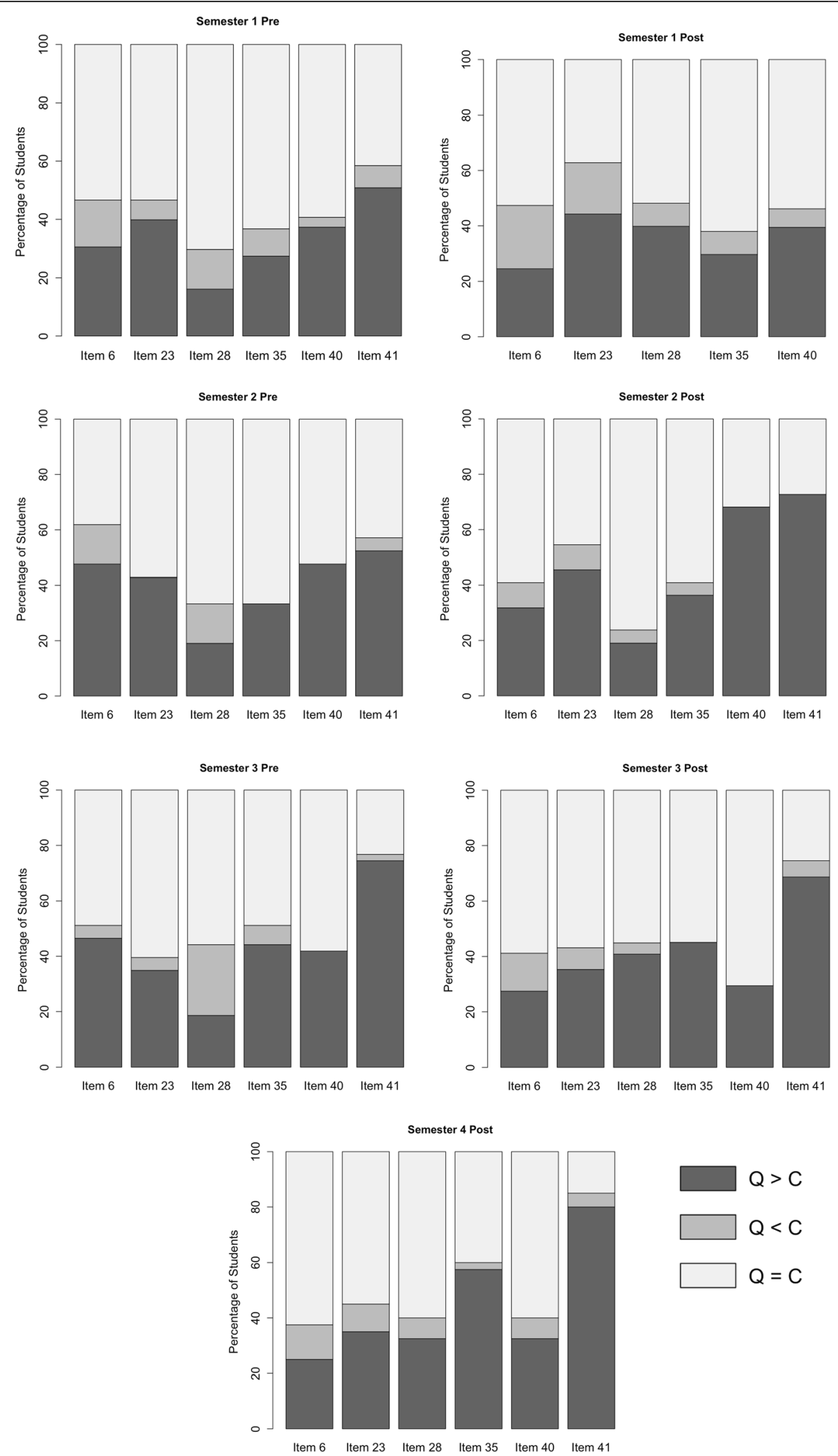

Fig. 1 (See legend on next page.) 
(See figure on previous page.)

Fig. 1 Classical/quantum splits on the six bifurcated CLASS items for the seven survey administrations. Each bar shows the percentages of students who did not split their response $(Q=C)$, split their responses with less agreement for quantum than classical $(Q<C$, i.e., quantumdisagree/classical-agree, quantum-disagree/classical-neutral, quantum-neutral/classical-agree), and split their responses with more agreement for quantum than classical $(\mathrm{Q}>\mathrm{C})$

quantum mechanics and not just to middle- and upper-division physics in general.

\section{Tara}

Responding to an open-ended prompt about her experience in the Modern Physics course, Tara spontaneously compared approaches to learning QM vs. classical physics: "Learning how to learn in quantum physics was kind of a challenge... With classical mechanics, you sort of have an intuition ... but with quantum mechanics nothing really makes sense. Like there's situations in which the potential energy is greater than the total energy... Quantum physics doesn't really follow the same trajectory as those courses do." Tara here implies that the trajectory of her earlier, classical courses connected to intuition, whereas "with quantum mechanics nothing makes sense"-leading to a different course trajectory.

\section{Fernando}

When asked about how physics and mathematics relate in QM, Fernando replied, "When it all comes down to it, if I don't understand what's going on conceptually, that's a little bit more difficult. Especially with this quantum stuff, I guess the conceptual work you need that mathematics behind it." We interpret "especially with this quantum stuff" to indicate a comparison with non-quantum stuff. Fernando is asserting that, in QM, the "conceptual work"-the intellectual work needed to make sense of the concepts-relies more ("especially") strongly on the underlying "mathematics behind it." This sentiment coheres with thoughts he expressed elsewhere in the interview.

\section{Eric}

Asked about the role of conceptual vs. mathematical reasoning in QM, Eric responds by drawing a distinction with classical physics courses: "I'd say the conceptual is less reliable now than it used to be, because in previous physics courses, the concepts were always intuitive... In quantum, it's more complicated, and less intuitive, so I'm less likely to trust my instincts on the concepts and more likely to trust the math."

\section{Zach}

This student's one spontaneous epistemological comparison comes very close to the border between "comparison" and "not a comparison." The interviewer asked, "What did you think about the focus group, now that it's kinda over. Um, yeah, what's your experience?" Zach responded, "I think it's cool. Like I definitely think like quantum mechanics is weird. I definitely think there's like merit in finding out how it's thought about and learned, just because it's so strange, like the ideas are so like really brand new. I have like, I had nothing in my head to attach it to before coming in. So, I mean, like yeah it's definitely like weird to like get your head going and wrap around some of the ideas..." Zach here does not explicitly compare the quantum weirdness he repeatedly mentions with any aspect of classical physics. We see evidence of an implicit comparison, though, when Zach calls QM "really brand new," with "nothing in my head to attach it to" before the course begins. These phrases connote a discontinuity with his previous experiences, including possibly prior physics courses. If we make the inference that the "strange," "weird" nature of quantum ideas constitutes part of the discontinuity, then Zach is saying that QM is new and weird compared to classical physics. We acknowledge, though, that this is a higher-level inference than we made in attributing a spontaneous epistemological comparison to the five previous students in this section.

For readers who do not agree with this inference, we note that Zach, responding to an open-ended prompt, spontaneously starts talking about the weirdness of quantum ideas. So, even if it is too much of a stretch to infer that he views quantum ideas as weirder than classical ideas, it is clear that quantum weirdness is salient to him.

In summary, of the seven interviewed students, five spontaneously expressed explicit epistemological comparisons and one spontaneously expressed epistemological views about QM that involved an implicit comparison with classical physics.

\section{Discussion}

\section{Summary and basic interpretation of results}

Before summarizing what this study shows, we reiterate our disclaimers about what it does not show. We cannot make claims about patterns in the differences between students' epistemological views about classical and quantum physics, because validated clusters of survey items would be needed to support such claims. Nor can we draw generalizations about students' reasoning in quantum mechanics or even students in the Modern Physics course from the interview analyses, because $N$ is small and because the spontaneous epistemological comparisons emerged in the context of an 
ongoing relationship between the research team and the interviewees.

By contrast, the "split" results indicate that most students do perceive epistemological differences to exist between classical and quantum physics, and the interview analyses suggest that at least some of these epistemological splits correspond to students' held views. So, when we talk about students' epistemologies of physics, we need to be careful about treating "physics" as homogeneous; these results show that a significant number of individual students display different approaches to knowledge in physics depending on the specific sub-discipline within physics. This exploratory study does not invalidate the CLASS, nor does it create an alternative survey instrument. Rather, it provides a proof of concept that students' epistemological views may differ by sub-domain of physics, and we argue that we should be attending to this domain specificity.

As discussed above, previous work had documented context dependence in students' epistemologies of physics. However, in that work, the contextual shifts associated with epistemological shifts corresponded to classroom vs. interview settings (Lising \& Elby, 2005), conceptual vs. procedural knowledge (Muis \& Gierus, 2014), the use of abstract ("philosophical") vs. deeply contextualized survey questions (Leach et al., 2000), conceptual cues (Gupta \& Elby, 2011), and instructional interventions that produced shifts in students' framing of their classroom activity (Rosenberg, Hammer, \& Phelan, 2006). What is new in this study, for PER and for personal epistemology research more broadly, is context dependence in students' epistemologies and expectations based on sub-discipline. The results of our exploratory study demonstrate the existence of students' epistemological splits between two sub-domains of physics-classical and quantum. We argue that, as a field, we should attend to this domain specificity of students' epistemological views. Further, we consider the possibility that these splits can reflect epistemological sophistication.

\section{What counts as epistemological sophistication?}

These results highlight an important interpretational issue about how to interpret the "split" results. Usually, CLASS responses and other epistemological survey responses are scored as favorable or unfavorable (or neutral). Applying this scoring procedure to the bifurcated survey results, one could conclude that students hold less favorable views about quantum physics than they do about classical physics. Specifically, from the post-semester results in Table 1 and Fig. 1 , one would conclude-to the extent that responses to individual survey items allow conclusions-that the students in our dataset who have completed a quantum physics class hold less sophisticated views about the utility of sense-making in quantum physics than they hold about the utility of sense-making in classical physics (CLASS \#23, \#40). One would also conclude that the students hold less sophisticated views about how quantum physics relates to the real world than they hold about how classical physics relates to the real world (CLASS \#35). In addition, one would conclude that they are more sophisticated about how quantum physics necessitates a rethinking of how the world works than they are about how classical physics necessitates a rethinking of how the world works (CLASS \#28).

We question this interpretation of our split results for both theoretical and empirical reasons.

\section{Theoretical argument: epistemological sophistication centers around nuanced contextualized judgments}

As discussed in previous literature on students' epistemologies of science (Elby \& Hammer, 2001; Elby, Macrander, \& Hammer, 2016; Sandoval, 2005), the default scoring scheme for epistemological surveys, counting some responses as more favorable than others, is not merely a matter of convenience or habit ("that's what everyone does!"). Such scoring schemes encode an assumption about the nature of epistemological expertise, that it involves holding certain favorable views at the expense of other, less favorable views. So, for instance, the coding and scoring schemes used in interview- and survey-based epistemology research encode the assumption that scientific knowledge should be viewed consistently: as tentative rather than certain, coherent rather than piecemeal, constructed by people rather than "read off" of nature or transmitted from authority, and so on (Elby \& Hammer, 2001).

An emerging consensus, however, holds that epistemological sophistication consists not of adherence to "blanket generalizations" (Elby \& Hammer, 2001), but to the ability and propensity to make contextualized judgments about knowledge-generating processes, coupled to criteria for monitoring and evaluating those processes and their results (Chinn, Rinehart, \& Buckland, 2014). For instance, it makes epistemological sense to treat "the Earth is round" as certain knowledge, and telephone numbers as transmitted from authority to recipients. It makes epistemological sense to treat the learning of complex concepts like Newton's second law as more "constructivist" than learning the value of the universal gravitational constant.

Applying this argument to classical vs. quantum physics, we could argue that students are sophisticated to display epistemological splits on our bifurcated surveyto treat classical and quantum physics as epistemologically different in certain ways. It is reasonable to view quantum concepts as less intuitive than classical concepts and therefore to rely more on mathematics and 
less on intuitive reasoning when solving problems. Along the same lines, it is reasonable to view quantum mechanics as requiring a deeper rethinking of how the world works than classical mechanics does; at least some physics experts would agree with these "split" views. Of course, epistemological expertise does not demand these splits; depending on one's interpretation of quantum mechanics, experience with quantum concepts, and so on, an expert could decide that classical and quantum mechanics are more epistemologically similar than different. So, we would expect some experts not to split. Our point is that there are likely sophisticated reasons for splitting and for not splitting, and hence the default interpretation of epistemological splits should not be that students possess greater epistemological sophistication about one subfield of physics than another.

\section{Empirical support for the epistemological reasonableness of classical-quantum splits}

The theoretical argument of the previous subsection makes predictions about the views of epistemologically sophisticated physicists: different physicists will display different epistemologies of quantum physics, likely leading to differences in their tendencies to express classicalquantum epistemological splits.

Accounts produced by historians and philosophers of quantum mechanics align with these predictions. Max Jammer (1989) and others have documented the lively debates among Bohr, Einstein, Heisenberg, and other early developers of QM about how to interpret quantum mechanics. The interpretational dilemmas and disagreements have not all been resolved (see, e.g., any issue of Foundations of Physics).

Preliminary results from another thread of the larger study also align with those predictions (Hoehn \& Finkelstein, 2018a). In that study, the CU co-authors administered the bifurcated survey to two instructors-one from the $\mathrm{CU}$ modern physics course and the other from the $\mathrm{CU}$ upper division quantum 1 course. On the quantum versions of the items, the two instructors' responses differed from one another on four of the six items. Furthermore, instructor 1 expressed classical-quantum splits on three of six items (two of these being "weak" splits between strongly disagree and disagree), and instructor 2 split on three of the items (one of these being a "weak" split). Obviously, we cannot generalize from these small$N$ results, but they suggest the likely value of a larger, partly interview-based study of experts' classicalquantum splits (and non-splits).

\section{Initial implications for instruction}

As we attend to how students think in domain-specific ways about the nature of learning and knowing, we ought to consider implications for not only how we measure impacts of our classes but also how we teach. The consistency of our "split" findings across multiple instructional contexts, for most of the bifurcated survey items, leads us to offer one thought about implications of this work for instruction. Although experts may disagree about what constitutes the right degree of classical-quantum splitting with respect to the value of conceptual reasoning (versus the need to "shut up and calculate"), we worry that quantum physics students are in danger of sliding too far away from conceptual reasoning. Students may internalize messages (intended or not) that quantum mechanics is a place to rely solely on mathematical calculation (Johansson, Andersson, SalminenKarlsson, \& Elmgren, 2016; Marshman \& Singh, 2015; Mermin, 1989) and that sense-making will not avail them here, which may impede the activation of the productive epistemological resources that they bring to classical physics. As instructors, we should not assume that students' "plug and chug" tendencies are entirely a "natural" epistemological response to quantum weirdness; we need to monitor ourselves and our students to avoid amplifying that tendency.

\section{Future directions}

As we know that instructors' epistemological views can be implicitly and explicitly reflected in their teaching (Linder, 1992; Marshall \& Linder, 2005; Tolhurst, 2007), and that faculty members' attitudes toward QM and thus their approaches to teaching QM vary (Dubson, Goldhaber, Pollock, \& Perkins, 2009; Siddiqui \& Singh, 2010), we will continue to have instructors of the courses for which we have student responses take the survey themselves. This will help us determine to what extent instructors' epistemological beliefs about QM are reflected in the student responses. It would also be interesting to conduct the bifurcated survey with a larger sample of expert physicists, to determine to what extent experts report splits and if there is an expert consensus on any of the bifurcated items.

We plan to continue using the bifurcated CLASS instrument (the six items included here as well as additional epistemology questions that ask only about $\mathrm{QM})$ to collect responses from more student populations in different pedagogical contexts. This will allow us to investigate the prevalence of epistemological splits and potentially the extent to which the classical/quantum splits are related to content differences versus pedagogical or other factors. In conducting studies of a broader scope, it may also be useful to conduct formal validation interviews with the bifurcated CLASS items.

Additional studies could involve bifurcating the survey on other content lines besides classical/quantum. Most students first encounter classical and quantum physics in introductory and upper-level physics courses, respectively. Therefore, exploring classical/quantum splits runs the risk of 
introductory vs. upper-level physics being a confounding factor (though our interview data show that this does not account for the full story). However, there are multiple subdomains of physics within the standard introductory physics curriculum, and our experience with teaching introductory physics suggests that students may take different epistemological approaches in "first-semester" and "second-semester" introductory physics. Future studies can investigate this directly.

Finally, further analysis of the data set in this paper will look at pre-post shifts on the bifurcated survey items, specifically addressing the question: Are the pre/post shifts in students' epistemological beliefs before and after a Modern Physics course different for quantum and classical physics?

\section{Conclusions}

This preliminary study demonstrates that a significant number of students taking modern physics and quantum mechanics (in a variety of settings) report different epistemological beliefs about classical physics and quantum physics, both before and after instruction in quantum physics. Specifically, students reported different beliefs in regard to the connection between (classical/quantum) physics and the real world, and the connection between mathematical and conceptual reasoning. From the student interview data, we observe ways that students make spontaneous epistemological comparisons between classical and quantum physics, suggesting that the quantitative results from the bifurcated survey represent a real phenomenon and not merely an artifact of the way the questions were posed. The interpretation of these results may not merely be about greater or lesser sophistication in each sub-domain of physics, but about identifying real differences in approach between sub-domains of the discipline (which may itself be an element of epistemological sophistication).

While this study was conducted in a physics context, similar investigations could be extended to other STEM disciplines. Other research on interactions between disciplines has already documented that students can display different attitudes to (e.g.) physics and biology (Gouvea, Sawtelle, \& Nair, 2019; Sawtelle \& Turpen, 2016), but future studies could also look at sub-domain specificity within other disciplines. The CLASS has been adapted for biology (Semsar, Knight, Birol, \& Smith, 2011) and chemistry (Adams, Wieman, Perkins, \& Barbera, 2008), and like the original physics version, the CLASS items that refer to "biology" and "chemistry" do not specify sub-domains of the disciplines. For example, our anecdotal experience with biology students, peer educators, and faculty suggests that students apply (and instructors expect them to apply) different epistemological resources in cell biology and in ecology. Our results from physics suggest questions that can be asked in other disciplines.

\section{Appendix}

Interview protocol

The questions below served as a guide for interviewers in the 30-minute semi-structured interviews with seven individual students from the CU Modern Physics course in Semester 1. Not all of the questions were asked in every interview. Question 3 asks students how the Modern Physics course changed the way they think about physics. If a student responded to this question by comparing epistemological aspects of classical and quantum physics, we did not code it as spontaneous due to the comparative nature of the question.

1. How did the semester go for you overall? (class and focus group)

2. What aspects of the course did you like or not like?

3. Has this course changed the way you think about physics? How?

4. How do you see the roles of calculation vs. conceptual reasoning in quantum mechanics?

5. What was your favorite thing (content area) you learned from class?

6. What was the most challenging thing for you in this class? (content-wise)

7. In the following situations, which of the interpretations do you feel like you are using? Why?

a. Double slit experiment (with beam of light, single photon, electrons)

b. Photoelectric effect

c. Electron transition in an atom

d. Aspect experiments with one/two beam splitters

e. Shining light on barrels to heat them

8. What did you think about the focus group?

9. What were the most/least productive aspects of the focus group for you?

10. Did your participation in this group affect your thinking in the class?

\section{Additional file}

Additional file 1: Quantitative results (DOCX $54 \mathrm{~kb}$ )

\section{Abbreviations}

CLASS: Colorado Learning Attitudes about Science Survey; CU: University of Colorado; FCl: Force Concept Inventory; MPEX: Maryland Physics Expectations Survey; PER: Physics education research; QM: Quantum mechanics;

UMD: University of Maryland

\section{Acknowledgements}

The authors thank Erin Ronayne Sohr, Kathleen Hinko, Doyle Woody, and Isaac Hanemann for their contributions to the collection and analysis of the 
data; Michelle Porcelli for initial conversations that led to the development of the split survey; Suzanne Brahmia, Eric Kuo, and Bethany Wilcox for helpful suggestions about statistics; the CU PER group for helpful discussions and feedback on the paper; course instructors for facilitating access to students; and all the anonymous students and instructors who took the surveys and participated in interviews.

\section{Authors' contributions}

BWD was involved in the study design, collected data at UMD, did the initial analysis of the quantitative data, drafted the first version of the manuscript, and took part in subsequent revisions. JRH collected data at CU, conducted some of the analysis of the quantitative data, conducted some of the interviews with students, was involved in the coding analysis of student interviews, and contributed substantially to the revisions of the manuscript at several different stages. AE co-wrote the literature review on student epistemologies and the qualitative methods and analysis. NDF contributed to study design, analysis, arguments, and contributed to the writing. AG contributed to the study design and the qualitative analysis methodology. All authors read and approved the final manuscript.

\section{Funding}

This work was supported by NSF-DUE grants 13-22734, 13-23129, and 1625797, and an NSF Graduate Research Fellowship. Publication of this article was funded by the George Mason University Libraries Open Access Publish ing Fund, the University of Colorado Boulder Libraries Open Access Fund, and the UMD Libraries' Open Access Publishing Fund.

\section{Availability of data and materials}

The datasets generated and analysed during the current study are not publicly available due to privacy concerns but are available from the authors (with student identifiers removed) on reasonable request.

\section{Ethics approval and consent to participate}

The study was approved by the Institutional Review Boards at both universities, and consent was obtained from all participants.

\section{Competing interests}

The authors declare that they have no competing interests.

\section{Author details}

'Department of Physics and Astronomy/STEM Accelerator Program, George Mason University, Fairfax, VA 22030, USA. ²Department of Physics, University of Colorado, Boulder, CO 80309, USA. ${ }^{3}$ Department of Teaching and Learning, Policy and Leadership, University of Maryland, College Park, MD 20742, USA. ${ }^{4}$ Department of Physics, University of Maryland, College Park, Maryland 20742, USA.

Received: 14 May 2019 Accepted: 27 August 2019

Published online: 17 September 2019

\section{References}

Adams, W. K., Perkins, K. K., Podolefsky, N. S., Dubson, M., Finkelstein, N. D., \& Wieman, C. E. (2006). New instrument for measuring student beliefs about physics and learning physics: The Colorado Learning Attitudes about Science Survey. Physical Review Special Topics - Physics Education Research, 2(1), 010101 https://doi.org/10.1103/PhysRevSTPER.2.010101.

Adams, W. K., Wieman, C. E., Perkins, K. K., \& Barbera, J. (2008). Modifying and validating the Colorado Learning Attitudes about science survey for use in chemistry. Journal of Chemical Education, 85(10), 1435 https://doi.org/10. 1021/ed085p1435.

Baily, C., \& Finkelstein, N. D. (2009). Development of quantum perspectives in modern physics. Physical Review Special Topics - Physics Education Research, 5(1), 010106 https://doi.org/10.1103/PhysRevSTPER.5.010106.

Baily, C., \& Finkelstein, N. D. (2010). Teaching and understanding of quantum interpretations in modern physics courses. Physical Review Special Topics Physics Education Research, 6(1), 010101 https://doi.org/10.1103/ PhysRevSTPER.6.010101

Bates, S. P., Galloway, R. K., Loptson, C., \& Slaughter, K. A. (2011). How attitudes and beliefs about physics change from high school to faculty. Physical Review Special Topics - Physics Education Research, 7(2), 020114 https://doi.org/10. 1103/PhysRevSTPER.7.020114.
Bhapkar, V. P. (1966). A Note on the equivalence of two test criteria for hypotheses in categorical data. Journal of the American Statistical Association, 61(313), 228 https://doi.org/10.2307/2283057.

Brewe, E., Kramer, L., \& O'Brien, G. (2009). Modeling instruction: Positive attitudinal shifts in introductory physics measured with CLASS. Physical Review Special Topics - Physics Education Research, 5, 013102 https://doi.org/10.1103/physrevstper.5.013102.

Chinn, C. A., Buckland, L. A., \& Samarapungavan, A. (2011). Expanding the dimensions of epistemic cognition: Arguments from philosophy and psychology. Educational Psychologist, 46(3), 141-167 https://doi.org/10.1080/00461520.2011.587722.

Chinn, C. A., Rinehart, R. W., \& Buckland, L. A. (2014). Epistemic cognition and evaluating information: Applying the AIR model of epistemic cognition. In Processing inaccurate information: Theoretical and applied perspectives from cognitive science and the educational sciences (pp. 425-453).

CU Boulder fall enrollment over time—By college—University of Colorado Boulder IR Tableau Public. (n.d.). Retrieved August 14, 2019, from https://public.tableau.com/ profile/university.of.colorado.boulder.ir\#!/vizhome/allcolleges/CollegeSummary

CU Boulder fall enrollment over time—By major-University of Colorado Boulder IR | Tableau Public. (n.d.). Retrieved August 14, 2019, from https://public.tableau.com/ profile/university.of.colorado.boulder.ir\#!/vizhome/allmajors/MajorSummary

Ding, L., Chabay, R., \& Sherwood, B. (2013). How do students in an innovative principle-based mechanics course understand energy concepts?: Assessing M\&l students' energy conceptions. Journal of Research in Science Teaching, 50(6), 722-747 https://doi.org/10.1002/tea.21097.

Docktor, J. L., \& Mestre, J. P. (2014). Synthesis of discipline-based education research in physics. Physical Review Special Topics - Physics Education Research, 10(2), 020119 https://doi.org/10.1103/PhysRevSTPER.10.020119.

Dreyfus, B. W., Elby, A., Gupta, A., \& Sohr, E. R. (2017). Mathematical sense-making in quantum mechanics: An initial peek. Physical Review Physics Education Research, 13(2), 020141 https://doi.org/10.1103/PhysRevPhysEducRes.13.020141.

Dubson, M., Goldhaber, S., Pollock, S., \& Perkins, K. (2009). Faculty disagreement about the teaching of quantum mechanics. AIP Conference Proceedings, 1179(1), 137-140 https://doi.org/10.1063/1.3266697.

EBAPS home. (n.d.). Retrieved April 25, 2018, from http://www2.physics.umd. edu/ elby/EBAPS/home.htm

Edmondson, K. M., \& Novak, J. D. (1993). The interplay of scientific epistemological views, learning strategies, and attitudes of college students. Journal of Research in Science Teaching, 30(6), 547-559 https://doi.org/10.1002/tea.3660300604.

Elby, A. (2011). Getting started with research on epistemologies and expectations. Reviews in PER, 2(1) Retrieved from http://www.per-central.org/items/detail. $\mathrm{cfm} ? \mathrm{ID}=10578$.

Elby, A., \& Hammer, D. (2001). On the substance of a sophisticated epistemology. Science Education, 85(5), 554-567 https://doi.org/10.1002/sce.1023.

Elby, A., Macrander, C., \& Hammer, D. (2016). Epistemic cognition in science. In J. A. Greene, W. A. Sandoval, \& I. Bråten (Eds.), Handbook of epistemic cognition (pp. 113-127). Routledge.

Gire, E., Jones, B., \& Price, E. (2009). Characterizing the epistemological development of physics majors. Physical Review Special Topics - Physics Education Research, 5(1), 010103 https:/doi.org/10.1103/PhysRevSTPER.5.010103.

Gouvea, J., Sawtelle, V., \& Nair, A. (2019). Epistemological progress in physics and its impact on biology. Physical Review Physics Education Research, 15(1), 010107 https://doi.org/10.1103/PhysRevPhysEducRes.15.010107.

Gray, K. E., Adams, W. K., Wieman, C. E., \& Perkins, K. K. (2008). Students know what physicists believe, but they don't agree: A study using the CLASS survey. Physical Review Special Topics - Physics Education Research, 4(2), 020106 https://doi.org/10.1103/PhysRevSTPER.4.020106.

Greene, J. A., Sandoval, W. A., \& Bråten, I. (2016). Handbook of Epistemic Cognition. Routledge.

Gupta, A., \& Elby, A. (2011). Beyond epistemological deficits: Dynamic explanations of engineering students' difficulties with mathematical sensemaking. International Journal of Science Education, 33(18), 2463-2488 https:// doi.org/10.1080/09500693.2010.551551

Hammer, D. (1994). Epistemological beliefs in introductory physics. Cognition and Instruction, 12(2), 151-183.

Hestenes, D., Wells, M., \& Swackhamer, G. (1992). Force concept inventory. The Physics Teacher, 30(3), 141-158 https://doi.org/10.1119/1.2343497.

Hoehn, J. R., \& Finkelstein, N. D. (2018a). Investigating and promoting epistemological sophistication in quantum physics. PERC 2017 Proceedings, 7-10. https://doi.org/10.1119/perc.2017.juried.002

Hoehn, J. R., \& Finkelstein, N. D. (2018b). Students' flexible use of ontologies and the value of tentative reasoning: Examples of conceptual understanding in three canonical topics of quantum mechanics. Physical Review Physics 
Education Research, 14(1), 010122 https://doi.org/10.1103/ PhysRevPhysEducRes. 14.010122.

Hoehn, J. R., Gifford, J. D., \& Finkelstein, N. D. (2019). Investigating the dynamics of ontological reasoning across contexts in quantum physics. Physical Review Physics Education Research, 15(1), 010124 https://doi.org/10.1103/ PhysRevPhysEducRes.15.010124.

Hofer, B. K. (2006). Domain specificity of personal epistemology: Resolved questions, persistent issues, new models. International Journal of Educational Research, 45(1-2), 85-95 https://doi.org/10.1016/j.jer.2006.08.006.

Hofer, B. K., \& Pintrich, P. R. (1997). The Development of epistemological theories: Beliefs About knowledge and knowing and their relation to learning. Review of Educational Research, 67(1), 88-140 https://doi.org/10.3102/ 00346543067001088.

Holm, S. (1979). A simple sequentially rejective multiple test procedure. Scandinavian Journal of Statistics, 6(2), 65-70.

Ireland, C. T., Ku, H. H., \& Kullback, S. (1969). Symmetry and marginal homogeneity of an $r \times r$ Contingency Table. Journal of the American Statistical Association, 64(328), 1323 https://doi.org/10.2307/2286071.

Jamieson, S. (2004). Likert scales: How to (ab)use them. Medical Education, 38(12), 1217-1218 https://doi.org/10.1111/j.1365-2929.2004.02012.x. Jammer, M. (1989). The conceptual development of quantum mechanics. Tomash

Johansson, A., Andersson, S., Salminen-Karlsson, M., \& Elmgren, M. (2016). "Shut up and calculate": The available discursive positions in quantum physics courses. Cultural Studies of Science Education, 1-22. https://doi.org/10.1007/ s11422-016-9742-8

Keefe, T. J. (1982). On the relationship between two tests for homogeneity of the marginal distributions in a two-way classification. Biometrika, 69(3), 683-684 https://doi.org/10.1093/biomet/69.3.683.

Leach, J., Millar, R., Ryder, J., \& Séré, M.-G. (2000). Epistemological understanding in science learning: The consistency of representations across contexts. Learning and Instruction, 10(6), 497-527. https://doi.org/10.1016/S0959-4752 (00)00013-X

Leinonen, R., Asikainen, M. A. \& Hirvonen, P. E. (2013). Overcoming students' misconceptions concerning thermal physics with the aid of hints and peer interaction during a lecture course. Physical Review Special Topics - Physics Education Research, 9(2), 020112 https://doi.org/10.1103/PhysRevSTPER.9.020112.

Linder, C. (1992). Is teacher-reflected epistemology a source of conceptual difficulty in physics? International Journal of Science Education, 14(1), 111-121.

Lising, L., \& Elby, A. (2005). The impact of epistemology on learning: A case study from introductory physics. American Journal of Physics, 73(4), $372 \mathrm{https}: / /$ doi. org/10.1119/1.1848115.

Madsen, A., McKagan, S. B., \& Sayre, E. C. (2015). How physics instruction impacts students' beliefs about learning physics: A meta-analysis of 24 studies. Physical Review Special Topics - Physics Education Research, 11, 010115.

Marshall, D., \& Linder, C. (2005). Students' expectations of teaching in undergraduate physics. International Journal of Science Education, 27(10), 1255-1268 https://doi.org/10.1080/09500690500153691.

Marshman, E., \& Singh, C. (2015). Framework for understanding the patterns of student difficulties in quantum mechanics. Physical Review Special Topics - Physics Education Research, 11(2), 020119 https:/doi.org/10.1103/PhysRevSTPER.11.020119.

Mason, A., \& Singh, C. (2010). Surveying graduate students' attitudes and approaches to problem solving. Physical Review Special Topics - Physics Education Research, 6(2), 020124 https://doi.org/10.1103/PhysRevSTPER.6.020124.

Maxwell, A. E. (1970). Comparing the classification of subjects by two independent judges. The British Journal of Psychiatry, 116(535), 651-655.

McCaskey, T. L. (2009). Comparing and contrasting different methods for probing student epistemology and epistemological development in introductory physics (University of Manyland). Retrieved from http:/drum.lib.umd.edu/handle/1903/9824

McCaskey, T. L., \& Elby, A. (2005). Probing Students' epistemologies using split tasks. AIP Conference Proceedings, 790(1), 57-60 https:/doi.org/10.1063/1.2084700.

McKagan, S. B., Perkins, K. K. \& Wieman, C. E. (2010). Design and validation of the quantum mechanics conceptual survey. Physical Review Special Topics - Physics Education Research, 6(2), 020121 https:/doi.org/10.1103/PhysRevSTPER.6.020121.

McNemar, Q. (1947). Note on the sampling error of the difference between correlated proportions or percentages. Psychometrika, 12(2), 153-157 https:// doi.org/10.1007/BF02295996.

Mermin, N. D. (1989). What's wrong with this pillow? Physics Today, 42(4), 9-11 https://doi.org/10.1063/1.2810963.

Muis, K. R., Bendixen, L. D., \& Haerle, F. C. (2006). Domain-Generality and domainspecificity in personal epistemology research: Philosophical and empirical reflections in the development of a theoretical framework. Educational Psychology Review, 18(1), 3-54 https://doi.org/10.1007/s10648-006-9003-6.
Muis, K. R., \& Gierus, B. (2014). Beliefs About knowledge, knowing, and learning: differences across knowledge types in physics. The Journal of Experimental Education, 82(3), 408-430 https://doi.org/10.1080/00220973.2013.813371.

Norman, G. (2010). Likert scales, levels of measurement and the "laws" of statistics. Advances in Health Sciences Education, 15(5), 625-632 https://doi. org/10.1007/s10459-010-9222-y.

Perkins, K. K. (2006). Towards characterizing the relationship between students' interest in and their beliefs about physics. AIP Conference Proceedings, 818, 137-140. https://doi.org/10.1063/1.2177042

Perkins, K. K., Adams, W. K., Pollock, S. J., Finkelstein, N. D., \& Wieman, C. E. (2005). Correlating student beliefs with student learning using the Colorado learning attitudes about science survey. AIP Conference Proceedings, 790, 61.

Redish, E. F., Saul, J. M., \& Steinberg, R. N. (1998). Student expectations in introductory physics. American Journal of Physics, 66(3), 212 https://doi.org/10.1119/1.18847.

Rodríguez, L., \& Cano, F. (2006). The epistemological beliefs, learning approaches and study orchestrations of university students. Studies in Higher Education, 31(5), 617-636 https://doi.org/10.1080/03075070600923442.

Rosenberg, S., Hammer, D., \& Phelan, J. (2006). Multiple epistemological coherences in an eighth-grade discussion of the rock cycle. Journal of the Learning Sciences, 15(2), 261-292 https://doi.org/10.1207/s15327809j/s1502_4.

Sandoval, W. A. (2005). Understanding students' practical epistemologies and their influence on learning through inquiry. Science Education, 89(4), 634-656 https://doi.org/10.1002/sce.20065.

Sawtelle, V., \& Turpen, C. (2016). Leveraging a relationship with biology to expand a relationship with physics. Physical Review Physics Education Research, 12(1), 010136 https://doi.org/10.1103/PhysRevPhysEducRes.12.010136.

Semsar, K., Knight, J. K., Birol, G., \& Smith, M. K. (2011). The Colorado learning attitudes about science survey (CLASS) for use in biology. CBE-Life Sciences Education, 10(3), 268-278 https://doi.org/10.1187/cbe.10-10-0133.

Siddiqui, S., \& Singh, C. (2010). Surveying instructors' attitudes and approaches to teaching quantum mechanics. AIP Conference Proceedings, 1289(1), 297-300 https://doi.org/10.1063/1.3515227.

Sohr, E. R., Gupta, A., \& Elby, A. (2018). Taking an escape hatch: Managing tension in group discourse. Science Education, 102(5), 883-916 https://doi. org/10.1002/sce.21448.

Stewart, J., Griffin, H., \& Stewart, G. (2007). Context sensitivity in the force concept inventory. Physical Review Special Topics - Physics Education Research, 3(1), 010102 https://doi.org/10.1103/PhysRevSTPER.3.010102.

Stuart, A. (1955). A test for homogeneity of the marginal distributions in a two-way CLASSIFICATION. Biometrika, 42(3/4), 412 https://doi.org/10.2307/2333387.

Sun, X., \& Yang, Z. (2008). Generalized McNemar's test for homogeneity of the marginal distributions. SAS Global Forum 2008 Proceedings. Presented at the SAS Global Forum 2008, San Antonio, Texas. Retrieved from https:/works.bepress.com/zyang/17/

Tolhurst, D. (2007). The influence of learning environments on students' epistemological beliefs and learning outcomes. Teaching in Higher Education, 12(2), 219-233 https://doi.org/10.1080/13562510701191992.

Tutorials on thinking about quantum entities (n.d.) https://www.physport.org/ methods/method. $\mathrm{cfm}$ ? G=quantum entities

UMD Undergraduate Student Profile. (2018). Retrieved from https://www.irpa. umd.edu/CampusCounts/Enrollments/stuprofile_allug.pdf

\section{Publisher's Note}

Springer Nature remains neutral with regard to jurisdictional claims in published maps and institutional affiliations. 